E D I T O R I A L S

\title{
Oral Desensitization to Peanuts
}

\author{
Michael R. Perkin, Ph.D.
}

Once a peanut allergy develops, advice has historically been simple: lifelong complete avoidance is needed to prevent systemic allergic reactions, some of which could be fatal. However, over the past decade, a series of case reports and small studies have shown that the systematic introduction of tiny amounts of peanut allergen, followed by gradual increases in dose, could prevent or attenuate systemic reactions..$^{1-4}$ The concept gained traction when a group in Cambridge, United Kingdom, found that $12 \%$ defatted peanut flour could induce desensitization in children. $^{5}$

Vickery and colleagues now present in the Journal $^{6}$ the results of a randomized, controlled trial involving approximately 550 participants with peanut allergy. The trial used a Good Manufacturing Process-produced 12\% defatted peanut flour preparation, known as AR101, as the allergen. For the peanut challenges at screening and trial exit, the authors chose to focus on the final tolerated dose (i.e., the dose that could be ingested without dose-limiting symptoms). In reality, families are interested in how much peanut their child can be exposed to in one meal without it inducing symptoms, not the final dose. Furthermore, peanuts vary in size and therefore in protein content, so translating the doses of peanut protein that were used in the trial to the equivalent in actual peanuts is difficult. In this editorial, I have used the conversion factor that was used by Vickery and colleagues - that is, that one peanut kernel contains $300 \mathrm{mg}$ of peanut protein (in contrast to the Cambridge group, which estimated that one peanut kernel contains
$160 \mathrm{mg}$ of peanut protein). In the primary analysis population of children and adolescents 4 to 17 years of age, after 1 year of treatment with AR101, before which they could consume less than half a peanut, two thirds of them could consume a cumulative dose of approximately four peanuts, whereas in the control group only 1 in 25 participants could consume this amount. In the 56 participants older than 17 years of age, no effect of AR101 treatment was shown.

Desensitization was not easy on the patients. Side effects during the intervention period that led to withdrawal from the trial occurred in $11.6 \%$ of the participants in the active-drug group and in $2.4 \%$ of those in the control group. This is not something to start at home. Epinephrine was used by $14.0 \%$ of the participants in the active-drug group as a result of reactions to treatment. The longer-term side effects of sustained consumption of an allergen to which the body has produced IgE antibodies remain unknown. Current thinking has focused on eosinophilic disease, such as eosinophilic esophagitis, but surveillance and follow-up will be crucial.

The major concern regarding immunotherapy is that the allergen tolerance that is induced will be temporary and lost if regular consumption ceases. Neither the Cambridge group, nor the investigators in this trial, have attempted to establish the duration for which allergen tolerance is maintained in the absence of ongoing consumption. Sustained unresponsiveness was claimed in an immunotherapy trial conducted by Tang et al., ${ }^{7}$ which also used peanut flour, but the median duration of cessation of consumption was only 
2.3 weeks - a period that would be better described as a brief interruption in therapy rather than as sustained cessation. Hence, sustained, potentially lifelong, regular consumption may be needed to maintain allergen tolerance. Most parents would see the regular consumption of a few peanuts by their child as a very small price to pay to keep the potential threat of systemic anaphylaxis at bay.

For the one third of participants who did not tolerate the cumulative dose of approximately four peanuts during the exit challenge, questions remain as to what role, if any, adjunctive therapy with, for example, anti-IgE therapy, epicutaneous immunotherapy, or probiotics might have in helping these persons benefit from oral desensitization. Once the increasing-dose phase is complete, maintenance treatment should continue with actual peanuts, as was offered by the Cambridge group when they initially investigated the efficacy and safety of their then-new oral immunotherapy protocol, which allowed participants the choice of receiving their maintenance immunotherapy as actual peanuts instead of as peanut flour. ${ }^{8}$

The clinical value of AR101 will be to allow the initiation of peanut immunotherapy with a product that reliably contains the tiny initial quantities of peanut that are required to safely launch oral desensitization. The lowest-dose capsules of AR101 contain $0.5 \mathrm{mg}$ and $1 \mathrm{mg}$ of peanut protein. In the absence of a product such as AR101, it is extremely difficult to administer such a small amount of allergen to a patient on a consistent basis. The Cambridge group used microscales and issued the doses of peanut flour in vials. However, errors regarding the initial doses during the increasing-dose phase would seem to be a more likely occurrence if allergy treatment centers all measured their own doses of peanut flour rather than using a carefully manufactured product. Furthermore, the issuing of peanut flour to a patient with peanut allergy may result in the peanut being deemed an unlicensed medicinal product by regulatory organizations in some countries. ${ }^{9}$ Once a product such as AR101 appears, such regulators will insist that a licensed product be used when it is available, thus preventing the ongoing use of peanut flour itself.

AR101 and other, similar products such as CA002, which is being developed by the Cambridge group, would therefore appear to have a role in initial dose escalation. The potential market for these products is believed to be billions of dollars. ${ }^{10}$ It is perhaps salutary to consider that in the study conducted by the Cambridge group, children underwent desensitization with a bag of peanut flour costing peanuts.

Disclosure forms provided by the author are available with the full text of this editorial at NEJM.org.

From the Population Health Research Institute, St. George's, University of London, London.

This editorial was published on November 18, 2018, at NEJM.org.

1. Mansfield L. Successful oral desensitization for systemic peanut allergy. Ann Allergy Asthma Immunol 2006;97:266-7.

2. Patriarca G, Nucera E, Pollastrini E, et al. Oral rush desensitization in peanut allergy: a case report. Dig Dis Sci 2006;51: 471-3.

3. Jones SM, Pons L, Roberts JL, et al. Clinical efficacy and immune regulation with peanut oral immunotherapy. J Allergy Clin Immunol 2009;124(2):292-300, 300.e1.

4. Clark AT, Islam S, King Y, Deighton J, Anagnostou K, Ewan PW. Successful oral tolerance induction in severe peanut allergy. Allergy 2009;64:1218-20.

5. Anagnostou K, Islam S, King Y, et al. Assessing the efficacy of oral immunotherapy for the desensitisation of peanut allergy in children (STOP II): a phase 2 randomised controlled trial. Lancet 2014;383:1297-304.

6. The PALISADE Group of Clinical Investigators. AR101 oral immunotherapy for peanut allergy. N Engl J Med 2018;379:19912001.

7. Tang ML, Ponsonby AL, Orsini F, et al. Administration of a probiotic with peanut oral immunotherapy: a randomized trial. J Allergy Clin Immunol 2015;135(3):737-44.e8.

8. Anagnostou K, Clark A, King Y, Islam S, Deighton J, Ewan P. Efficacy and safety of high-dose peanut oral immunotherapy with factors predicting outcome. Clin Exp Allergy 2011;41:127381.

9. The human medicines regulations 2012: regulation 2 . Norwich, United Kingdom: The Stationery Office (http://www .legislation.gov.uk/uksi/2012/1916/regulation/2/made).

10. GlobalData Healthcare. Peanut allergy market set for remarkable growth to 2027. Pharmaceutical Technology. July 4, 2018 (https://www.pharmaceutical-technology.com/comment/ peanut-allergy-market-set-remarkable-growth-2027/).

DOI: 10.1056/NEJMe1813314

Copyright @ 2018 Massachusetts Medical Society. 